\title{
Theranostic Nanoparticles: A Recent Breakthrough in Nanotechnology
}

\section{Narayan Bhattarai ${ }^{1 *}$ and Shanta R. Bhattarai ${ }^{2}$}

${ }^{1}$ Department of Chemical, Biological and Bioengineering, North Carolina A\&T State University, Greensboro, NC, USA

${ }^{2}$ Department of Radiation Oncology, The University of Texas MD Anderson Cancer Center, Houston, TX, USA

As a revolutionary new $21^{\text {st }}$ century technology, nanotechnology is starting to impact almost every aspect of society. Disease diagnosis and treatment is one of the highest impact areas where nanotechnology has excellent potential and promise [1,2]. Nanotechnology is also aiding in the formation of molecular systems that designed to be noticeably similar to living systems, which could be the basis for the regeneration of body parts that are currently lost due to infection, accident, or disease [3]. The National Cancer Institute, for instance, has created the Alliance for Nanotechnology in Cancer in the hope that investments in biomedical nanotechnology, such as nanomedicine, will lead to breakthroughs in terms of detecting, diagnosing, and treating various forms of cancer. Nanotechnology is already moving from being used as a passive structure in applications such as in cosmetics and sunscreens to active structures in clinical applications such as pharmaceuticals (targetable "smart drugs"). In the present context, nanotechnology is almost paronymous with nanoparticles, an umbrella term that refers to particles at dimensions of roughly about $100 \mathrm{~nm}$ or less. Integration of therapeutic compounds into nanoparticles with diagnostic agents refers to the theranostic nanoparticles (imaging and therapy). Examples of such nanotechnology products approved by the Food and Drug Administration (FDA) for human applications are based on iron oxide, gold, protein, liposomes and synthetic polymers [4,5].

In contract to traditional macroscopic materials, nanomaterials have high ratio of surface area to volume as well as tunable optical, electronic, magnetic, and biologic properties, they can be engineered to have different sizes, shapes, chemical compositions, and surface chemical characteristics $[4,5]$. The field of "theranostic nanoparticles" aims to use these properties and physical characteristics of nanomaterials for the diagnosis and treatment of diseases at the molecular level. For example, in cancer nanotheranostics, nanoparticles are functionalized by combining imaging and therapeutic agents to interact with cancer cells at the molecular level in such a way that the functionalized combo particles can significantly improve the effectiveness and specificity of therapy to cancers that are currently difficult to treat.

Surface functionalization of theranostic nanoparticles is one of the serious technological hurdles specially to maintain their dispersity, biocompatibility and biostability in physiological condition. Nanoparticles have high surface energy and tend to agglomerate and adsorb plasma proteins in body. Agglomerated nanoparticles are easily recognized by body's immune system and can be very toxic to body tissues. Therefore, a robust surface functionalization method is most important steps to increase particle dispersity and colloidal stability. Creating a water soluble polymer based non-fouling surface on the nanoparticles surface has been found as one of the most successful techniques to prevent the particle agglomeration, prolong the particle circulation time in the blood, and enhance the particle penetration across the biological barriers such as blood brain barriers [6]. The polymer shell also provides active sites for the conjugation of targeting ligands and diagnostic fluorophores offering nanoparticles specificity to diseased cells and an optical imaging visualization, respectively.

In addition, there is growing speculation about possible nanomaterial toxicity on the basis of in vitro cell-culture studies and in vivo animal studies. To translate these applications into clinical use, researchers must optimize the nanomaterials, beginning with smallanimal models and scaling up to nonhuman primate models - a process that requires relatively long time. These studies should provide a solid foundation for the long-term advancement of the theranostic nanoparticles into an effective new area in clinical medical practice. In order to fully realize such promises of nanotechnology in medicine, sharing the successes and challenges as well as disseminating the research findings in broader audience focused peer-reviewed journals, conferences and workshops are also equally important.

\section{Acknowledgements}

Authors would like to acknowledge Engineering Research Center for Revolutionizing Metallic Biomaterials (ERC-RMB) and Nanotechnology Undergraduate Education (NUE) form NSF for funding support.

\section{References}

1. Nel AE, Mädler L, Velegol D, Xia T, Hoek EM, et al. (2009) Understanding biophysicochemical interactions at the nano-bio interface. Nat Mater 8: 543557.

2. Service RF (2005) Materials and biology. Nanotechnology takes aim at cancer Science 310: 1132-1134

3. Dvir T, Timko BP, Kohane DS, Langer R (2011) Nanotechnological strategies for engineering complex tissues. Nat Nanotechnol 6: 13-22.

4. Xia Y, Xiong Y, Lim B, Skrabalak SE (2009) Shape-controlled synthesis of metal nanocrystals: simple chemistry meets complex physics. Angew Chem Int Ed Engl 48: 60-103.

5. Davis ME, Chen ZG, Shin DM (2008) Nanoparticle therapeutics: an emerging treatment modality for cancer. Nat Rev Drug Discov 7: 771-782.

6. Kievit FM, Veiseh O, Bhattarai N, Fang C, Gunn JW, et al. (2009) PEI-PEGchitosan copolymer coated iron oxide nanoparticles for safe gene delivery: synthesis, complexation, and transfection. Adv Funct Mater 19: 2244-2251.

*Corresponding author: Narayan Bhattarai, Department of Chemical, Biological and Bioengineering, North Carolina A \& T State University, McNair 326, Greensboro, NC USA, E-mail: nbhattar@ncat.edu

Received August 24, 2012; Accepted August 24, 2012; Published August 26 , 2012

Citation: Bhattarai N, Bhattarai SR (2012) Theranostic Nanoparticles: A Recent Breakthrough in Nanotechnology. J Nanomed Nanotechol 3:e114. doi:10.4172/2157-7439.1000e114

Copyright: (c 2012 Bhattarai N, et al. This is an open-access article distributed under the terms of the Creative Commons Attribution License, which permits unrestricted use, distribution, and reproduction in any medium, provided the original author and source are credited. 